\title{
Über die Messung der Photosynthese in Carbonat-Bicarbonat-Gemischen
}

\author{
Von Otto Warburg, Horst Geleick und Klaus Briese \\ Aus dem Kaiser-Wilhelm-Institut für Zellphysiologie, Berlin-Dahlem \\ (Z. Naturforschg. 7 b, 141-144 [1952]; eingegangen am 16. Januar 1952)
}

\begin{abstract}
Weiterentwicklung der Versuchstechnik zur Messung der Photosynthese mit der Ein-Gefäßmethode und Hinweis auf einige Fehlerquellen.
\end{abstract}

$S_{\mathrm{s}}$ eit 1918 die Carbonat-Bicarbonat-Gemische zur Manometrie der Photosynthese vorgeschlagen wurden ${ }^{1}$, sind die meisten Messungen der Photosynthese in derärtigen Gemischen ausgeführt worden, und zwar vorwiegend in dem Gemisch Nr. 9, das aus $85 \mathrm{Vol}$. $m / 10-\mathrm{NaHCO}_{3}$ und $15 \mathrm{Vol}$. $m / 10-\mathrm{Na}_{2} \mathrm{CO}_{3}$ besteht. $p_{\mathrm{H}}$ bei $20^{\circ}$ ist etwa 9,4. Der Kohlensäuredruck, mit dem das Gemisch bei $20^{\circ}$ im Gleichgewicht ist, beträgt etwa $0,22 \%$ einer Atmosphäre und wird bei Verbrauch oder Entwicklung von Kohlensäure durch die Reaktionen

$$
2 \mathrm{NaHCO}_{3} \rightleftarrows \mathrm{Na}_{2} \mathrm{CO}_{3}+\mathrm{H}_{2} \mathrm{O}+\mathrm{CO}_{2}
$$

konstant gehalten.

1939 begann Emers on ${ }^{2}$ das Gemisch Nr. 9 zur Messung von Energieausbeuten bei der Photosynthese zu benutzen und fand, daß in grünen Algen etwa 11 Lichtquanten absorbiert werden müssen, damit 1 Molekül Sauerstoff entwickelt wird. Seitdem vertraten Emerson und mit ihm Franck ${ }^{3}$, Gaf fro $n^{3}$ und Rabin owit $\mathrm{ch}^{4}$ die Auffassung, daß die Ausbeute $1 / 11=0,09$ Mole Sauerstoff pro Mol absorbierter Lichtquanten die maximal bei der Photosynthese erreichbare Ausbeute sei. Niemals jedoch ist von ihnen begründet worden, warum gerade in dem Carbonatgemisch Nr. 9 die Ausbeute die maximale _sein sollte.

Wie wir vor kurzem mitgeteilt haben ${ }^{5}$, läßt sich durch geringfügige Änderungen des Gemischs Nr. 9 erreichen, daß unter sonst gleichen methodischen Bedingungen; insbesondere mit der 1-Gefäßmethode, ein Quantenbedarf von 3,5 anstatt 11 gefunden wird; daß also in dem neuen Gemisch etwa das 3-fache der

1 O. W a r burg, Biochem. Z. 100, 230 [1919].

2 R. Emerson u. Ch. M. Lew is, Amer. J. Bot. 28, 789 [1941].

3 James Franck u. Hans Gaffron, Advances in Enzymology 1, 199 [1941].

${ }_{4}$ Eugène Ra bi now it s c h, Photosynthesis. Tl. II. Interscience Publishers, New York 1951. photochemischen Ausbeute gefunden wird, die Emerson in dem Gemisch Nr. 9 gefunden hatte.

Unser neues Gemisch besteht aus 95 Vol. $m / 5$ $\mathrm{NaHCO}_{3}$ und 5 Vol. $m / 5-\mathrm{Na}_{2} \mathrm{CO}_{3}$. Bei $20^{\circ}$ ist $p_{\mathrm{H}} 8,8$. Der Kohlensäuredruck, mit dem das neue Gemisch im Gleichgewicht ist, beträgt bei $20^{\circ}$ etwa $2 \%$ einer Atmosphäre, ist also rund 10 -mal so groß wie der Kohlensäuredruck des Gemischs Nr. 9. Der höhere Kohlensäuredruck ist die Ursache der besseren Ausbeute in dem neuen Gemisch; denn auch in saurer Kulturlösung findet man bei $2 \%$ Kohlensäure eine viel bessere Ausbeute als bei $0,2 \%$. Die niedrige Ausbeute in dem Carbonatgemisch Nr. 9 ist also die niedrige Ausbeute, die zu dem niedrigen Kohlensäuredruck von $0,2 \%$ gehört, aber nicht die maximale Ausbeute.

Bei der Fortsetzung unsrer Versuche über Photosynthese in Carbonatgemischen sind wir auf Unregelmäßigkeiten gestoßen, die wahrscheinlich mit der Diffusion von Bicarbonat in die Zellen zusammenhängen. Wir beschreiben im folgenden diese Unregelmäßigkeiten und teilen mit, wie man sie im wesentlichen vermeiden kann.

Photochemische Ausbeuten sind früher in Carbonatgemischen, wegen ihrer unphysiologischen Zusammensetzung, von uns nicht gemessen worden ${ }^{6}$. Trotzdem schien uns nunmehr die Ausarbeitung der Carbonatmethode notwendig. Allerdings bleibt sie der heute fast zur Vollkommenheit entwickelten 2-Gefäßmethode unterlegen, mit der man die Sauerstoffentwicklung wie die Kohlensäurefixierung unter völlig physiologischen Bedingungen für beliebig lange Zeiten bestimmen kann.

5 O. W a rburg, H. Ge le ick u. K. Briese, Z. Naturforschg. 6 b, 285 [1951].

6 "It would never have occurred to us to measure the most delicate of all biochemical prozesses, the conversion of light energy into chemical energy, in a medium, in which the very survival of the cells seems remarkable“ (O. W a r bu r g, Amer. J. Bot. 35, 194 [1948]). 


\section{Die Züchtung der Zellen}

ist in Abb. 1 veranschaulicht. Sie wird in einem hellen Nordzimmer vorgenommen, in dem die Kulturen neben dem Licht der 200-Watt-Metallfadenlampe reichlich Tageslicht erhalten. Die Aussaat beträgt $30 \mathrm{cmm}$ Zellen pro Kolben, die Ernte nach $48 \mathrm{Stdn}$. 500 bis $800 \mathrm{cmm}$ Zellen. Das Gas (5 $\mathrm{CO}_{2}$-Luft) wird so schnell durch die Kolben geleitet, daß die Zellen nicht sedimentieren. Die Kulturlösung hat die $\mathrm{Zu}$ sammensetzung

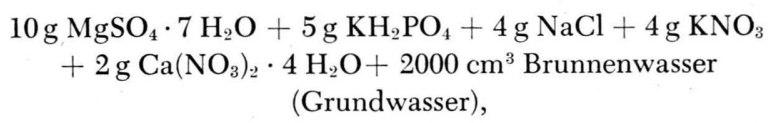

mit $n / 1$-Schwefelsäure auf $p_{\mathrm{H}} 4,6$ angesäuert. Vor Gebrauch werden Schwermetallsalze zugesetzt, und zwar in $2000 \mathrm{~cm}^{3}$

$$
\begin{aligned}
12 \mathrm{mg} \mathrm{FeSO} & \cdot 7 \mathrm{H}_{2} \mathrm{O}+0,12 \mathrm{mg} \mathrm{ZnSO}_{4} \cdot 7 \mathrm{H}_{2} \mathrm{O} \\
& +0,12 \mathrm{mg} \mathrm{\textrm {MSO } _ { 4 }} \cdot 4 \mathrm{H}_{2} \mathrm{O} .
\end{aligned}
$$

Calcium, Zink und Mangan haben wir auf Rat von Herrn Professor Pirs on, Marburg, zugesetzt.

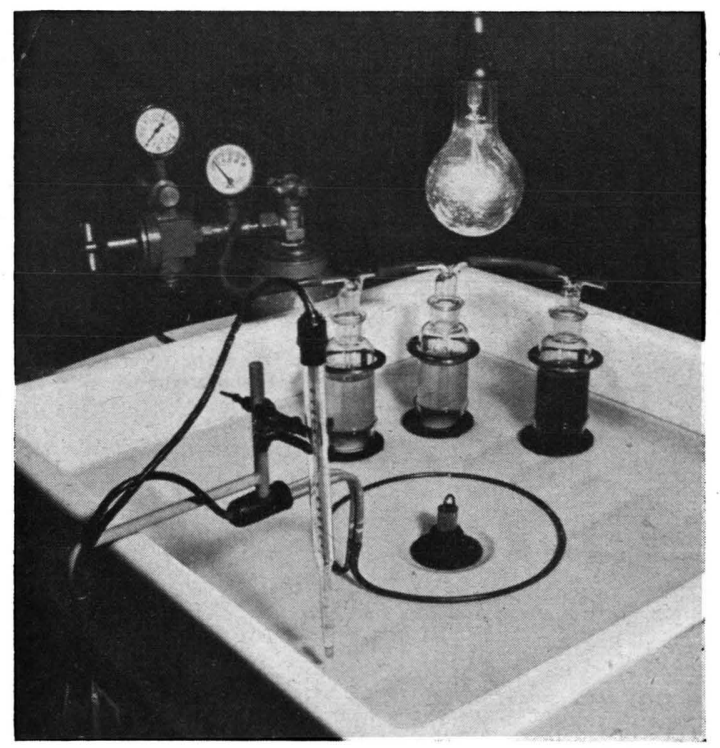

Abb. 1. Die Züchtung der Zellen.

Die Züchtungskolben haben einen Inhalt von $300 \mathrm{~cm}^{3}$ und werden mit $250 \mathrm{~cm}^{3}$ Flüssigkeit gefüllt. Die Kolben ragen zu etwa ${ }^{2} / 3$ aus dem Wasser heraus. Als Kontaktthermometer verwenden wir solche von Richter \& $\mathrm{W}$ i e s e in Berlin. Temperatur des Wassers $25^{\circ}$.

7 R. Emerson u. Ch. M. Lewis, Amer. J. Bot. 30, 165 [1943].
Wenn die Zellen zentrifugiert werden, sei es zur Weiterzüchtung, sei es zur Messung der Photosynthese, so werden Zentrifugiergläser mit breiten Böden benutzt, und es wird aus niedriger Schicht nur etwa $1^{1 / 2}$ Min. mit nur 500-facher Schwerkraft zentrifugiert. Die Zellen müssen so lccker am Boden der Gefäße liegen, daß sie beim Aufgießen von Flüssigkeit sofort aufgewirbelt werden.

2. Messungen in $\mathrm{Carbonatgem}$ is ch Nr. 9

Nach Emersons Vorschrift ${ }^{7}$ wird Chlorella 2-mal auf der Zentrifuge mit dem Carbonatgemisch Nr. 9 gewaschen und dann zur Messung in dem Gemisch suspendiert. Emersons Zelldichte war sehr groß, und zwar $300 \mathrm{cmm}$ Zellen pro Gefäß, um vollständige Lichtabsorption $\mathrm{zu}$ erzielen. Manometrie und Bolometrie entsprachen unseren Vorschriften ${ }^{8}$ von 1923: runde Gefäße, kreisförmige Bewegung, Differentialmanometer und Kathetometer. In Spektralgebieten, in denen die gelben Farbstoffe nicht absorbieren, fand Emerson einen Quantenbedarf von 11 pro Molekül entwickelten Sauerstoffs.

Zur Kontrolle haben wir die Versuche Emersons mit unseren neuen Methoden nachgeprüft. Wir benutzten einfache Manometer an Stelle der Differentialmanometer und horizontale Bewegung der Gefäße an Stelle der Kreisbewegung, wodurch das Schäumen der Zellsuspensionen vermieden wurde. Wir eliminierten die Atmung durch Vorkompensation mit weißem Licht; wir verdünnten die Zellen bis auf Kulturdichte und bestimmten in jedem Versuch die Lichtabsorption, die im Grün nur 25\% betrug, mit dem Transmissions-Actinometer ${ }^{9}$. Ein Quantenbedarf von 9-10 pro Molekül Sauerstoff wurde für die grüne Hg-Linie $546 \mathrm{~m} \mu$ gefunden. Die Differenz gegen Emersons 11 Quanten rührt daher, daß Emerson weder die Ultrarot-Absorption seiner BolometerQuarzscheibe noch den Lichtverlust auf dem Weg vom Bolometer durch den Thermostaten zum Manometergefäß berücksichtigte. Im groben aber können wir die Angaben Emersons soweit bestätigen, daß der Quantenbedarf in dem Carbonatgemisch Nr. 9, wenn man die Zellen vorher 2-mal darin wäscht, von der Größenordnung 10 ist.

3. Das neue Carbonatgemisch und sein Retentionsvermögen für Kohlensäure

Wie bereits in der Einleitung erwähnt, ist das neue Carbonatgemisch konzentrierter als das Ge-

8 O. W a r burg u. E. N e g e le in, Z. physik. Chem. 106, 191 [1923].

${ }^{9}$ D. B u rk u. O. W a r bu rg, Z. Naturforschg. 6 b, 12 [1951]. 
misch Nr. 9, und das Verhältnis Bicarbonat/Carbonat ist zugunsten des Bicarbonats verschoben. Von Basen prüften wir Natrium, Kalium, Lithium und Caesium und fanden Lithium und Caesium schädlich, Natrium mindestens ebensogut wie Kalium oder wie Gemische von Natrium und Kalium. Wurde das Verhältnis Bicarbonat/Carbonat über 95/5 gesteigert, so wurde die Retention der Kohlensäure zu klein; wurde die Salzkonzentration über $m / 5$ gesteigert, traten Schädigungen auf. $m / 595 / 5$ scheint also ein optimales Gemisch zu sein. Es wurde mit Wasser, das aus Quarz destilliert war, hergestellt.

Um die Retention zu bestimmen, gaben wir zunächst aus einer Ansatzbirne $0,2 \mathrm{~cm}^{3} n / 20$-Schwefelsäure in $7 \mathrm{~cm}^{3} n / 100-\mathrm{NaHCO}_{3}$, während der $\mathrm{CO}_{2}$ Druck im Gasraum 11\% einer Atmosphäre war $\left(v=21,82 \mathrm{~cm}^{3}, v_{\mathrm{F}}=7,2 \mathrm{~cm}^{3}, 20^{\circ}\right)$. Wir erhielten $+111 \mathrm{~mm}$ Brodie $=221 \mathrm{cmm} \mathrm{CO} \mathrm{CO}_{2}$. Wurde dann im gleichen Gefäß die gleiche Menge Schwefelsäure in $7 \mathrm{~cm}^{3}$ des Carbonatgemischs gegeben, während der Kohlensäuredruck im Gasraum 2,15\% einer Atmosphäre war, so wurden nur $10 \mathrm{~mm}$ Brodie $=19,8 \mathrm{cmm}$ $\mathrm{CO}_{2}$ erhalten. Die Retention $R$ durch $7 \mathrm{~cm}^{3}$ Carbonatgemisch betrug also

$$
R=\frac{221-19,8}{10}=20,1 \mathrm{cmm} \mathrm{CO} \text { pro mm Brodie }
$$

Wie wirkt diese Retention auf die manometrischen Ausschläge bei der Messung der Photosynthese?

Unsere Meßgefäße waren Kästchen mit den Abmessungen

$$
\begin{gathered}
v=16,59 \mathrm{~cm}^{3}, v_{\mathrm{F}}=7,0 \mathrm{~cm}^{3}, \\
\text { also bei } 20^{\circ}: k_{\mathrm{O}_{2}}=0,915 \text { und } k_{\mathrm{CO}_{2}}=1,503 \mathrm{~mm}^{2},
\end{gathered}
$$

wo $k_{\mathrm{CO}_{2}}$ für nicht retinierende Flüssigkeiten gilt. Ist aber die Retention $R=20$, so wird die Gefäßkonstante für Kohlensäure

$$
k_{\mathrm{CO}_{2}}=1,503+20=21,5 \mathrm{~mm}^{2}
$$

und $K_{02}$, wenn wir $\gamma=-1,1$ annehmen,

$$
\begin{aligned}
K_{\mathrm{O}_{2}}=\frac{k_{\mathrm{CO}_{2}} \cdot k_{\mathrm{O}_{2}}}{k_{\mathrm{CO}_{2}}+\gamma \cdot k_{\mathrm{O}_{2}}} & =\frac{21,5 \cdot 0,915}{21,5-1,0} \\
& =1,05 \cdot 0,915=1,05 k_{\mathrm{O}_{2}} .
\end{aligned}
$$

Etwa 5\% des entwickelten Sauerstoffs wird also wegen der nicht vollständigen Retention durch $\mathrm{Ab}$ sorption von Kohlensäure kompensiert, d. h., wir finden die wahre Druckzunahme des Sauerstoffs, wenn wir die beobachteten Drucke mit 1,05 multiplizieren. Das Praefix „korr“ im folgenden bedeutet, daß diese Korrektion angebracht worden ist.

Für das Gemisch $n / 5$ 95/5 ist also die Unvollständigkeit der Retention manometrisch fast bedeutungslos. Von anderen Carbonatgemischen, z. B. von $m / 5$ $98 / 2$, wird wesentlich weniger retiniert, und deshalb können solche Gemische für die 1-Gefäßmethode nicht empfohlen werden. Immer aber könnte unvollständige Retention nur bewirken, daß man bei Anwendung der 1-Gefäßmethode die Ausbeuten zu niedrig, niemals aber, daß man sie zu hoch findet.

\section{Messungen in dem neuen $\mathrm{Carbonatgemisch}$}

In den früher beschriebenen Versuchen mit dem neuen Carbonatgemisch wurden die Zellen vor $\mathrm{Zu}$ gabe des Gemischs 2-mal auf der Zentrifuge mit dest. Wasser gewaschen, um Niederschlagsbildung mit den Kultursalzen zu vermeiden. Wir haben inzwischen gefunden, daß das Waschen der Zellen gefährlich ist. Wäscht man vor dem Einbringen in das Carbonatgemisch mit Wasser, so sinkt die Ausbeute nach einigen Stunden, manchmal sogar sofort, ab. Wäscht man

\begin{tabular}{|c|c|}
\hline \multicolumn{2}{|c|}{ Zellen nicht gewaschen } \\
\hline \multicolumn{2}{|c|}{$\begin{array}{c}\text { Gemessen in } m / 595 / 5 \\
2 \text { Vol.- } \% \mathrm{CO}_{2} \text {-Luft }\end{array}$} \\
\hline \multicolumn{2}{|c|}{$1 / \varphi=h v / \mathrm{O}$} \\
\hline$J=6,23$ & 3,95 \\
\hline$J=6,23$ & 3,85 \\
\hline$J=6,23$ & 3,70 \\
\hline$J=9,55$ & 4,25 \\
\hline
\end{tabular}
aber, wie es Emerson bei seinen Versuchen mit dem Carbonatgemisch Nr. 9 tat, vorher mit dem Carbonatgemisch, so ist die Ausbeute im allgemeinen noch kurzlebiger und bricht im Lauf einiger Stunden vollständig zusammen, z. B.:

Zellen 1-mal mit $50 \mathrm{~cm}^{3}$ Carbonatgemisch $m / 5$ 95/5 gewaschen

Gemessen in $m / 5$ 95/5 2 Vol.- $\% \mathrm{CO}_{2}$-Luft

1. Stde. $J=6,23$

$1 / \varphi=h v / \mathrm{O}_{2}$

6,75

6,75

11,6

Photooxydation,

wo $J$ die eingestrahlte Quantenintensität in $\mathrm{cmm} / \mathrm{min}$ bedeutet. Die Wellenlänge war $546 \mathrm{~m} \mu$ und die Lichtabsorption betrug 21\% (Gefäß $v=16,59 \mathrm{~cm}^{3}$, $v_{\mathrm{F}}=7,0 \mathrm{~cm}^{3}, 20^{\circ}$ ). Die Atmung war durch weißes Licht kompensiert. Das Meßlicht wurde intermittierend in $1^{\prime} 1^{\prime}$ zugefügt.

Da sich weiterhin zeigte, daß beim Aufgießen des Carbonatgemischs auf Zellen, die aus Kulturlösung abzentrifugiert waren, keine Niederschläge entstanden, so war das Waschen der Zellen überflüssig und wurde unterlassen. 


\section{Zusatz von Kultursalzen $\mathrm{zu}$ dem Carbonatgemisch}

Nach den geschilderten Erfahrungen lag es nahe, den Carbonatgemischen Kultursalze zuzusetzen, um dadurch ihren unphysiologischen Zustand zu mildern. Wir fanden am günstigsten Zusatz von Magnesiumsulfat und Calciumnitrat in $1 / 100$ ihrer Konzentration in der Kulturflüssigkeit. Niederschläge traten dabei nicht auf; das Retentionsvermögen des Gemischs $m / 5$ 95/5 für Kohlensäure wurde durch die Zusätze nicht beeinflußt.

Auch das alte Carbonatgemisch Nr. 9 kann in seiner Wirkung durch Nichtwaschen und durch Salzzusätze verbessert werden. $\mathrm{Da}$ es aber wegen seines $\mathrm{zu}$ niedrigen Kohlensäuredrucks sehr schlechte Ausbeuten liefert, haben Versuche mit dem alten Gemisch heute kein Interesse mehr.

\section{Die Kohlensäurekurve}

Wenn, wie in dem Versuch des Abschnitts 4, die Ausbeute mit der Zeit absinkt, so ist es klar, daß der Kohlensäuredruck nicht die Ausbeute bestimmen kann. Denn der Kohlensäuredruck bleibt ja im zeitlichen Verlauf der Versuche konstant. Zu der Kurve, die die Abhängigkeit der Ausbeute vom Kohlensäuredruck darstellt ${ }^{5}$, ist also die fast selbstverständlich einschränkende Bedingung hinzuzufügen, daß während der Versuche keine irreversiblen Veränderungen erfolgen dürfen. Ist diese Bedingung nicht erfüllt, wie z. B. für Zellen, die mit Carbonatgemischen vorgewaschen worden sind, so kann man in verschiedenen Carbonatgemischen, bei sehr verschiedenen Kohlensäuredrucken, nahezu dieselben Ausbeuten erhalten, die aber nunmehr nicht nur bei niedrigen, sondern auch bei höheren Kohlensäuredrucken weit entfernt von den maximalen Ausbeuten sind. Dieses „Zusammenrücken“ der Ausbeuten trotz verschiedener Kohlensäuredrucke ist ein sicheres Zeichen progressiver Veränderungen innerhalb der Zellen, vermutlich einer Diffusion von Bicarbonat in die Zellen.

\section{Einige Beispiele}

1. Chlorella wurde aus der Kulturlösung zentrifugiert und mit dem Gemisch $m / 5$ 95/5 übergossen, dem auf $100 \mathrm{~cm}^{3} 5 \mathrm{mg} \mathrm{MgSO}_{4} \cdot 7 \mathrm{H}_{2} \mathrm{O}$ und $1 \mathrm{mg} \mathrm{Ca}\left(\mathrm{NO}_{3}\right)_{2} \cdot 4 \mathrm{H}_{2} \mathrm{O}$ zugesetzt waren. Gasraum 2,15\% Kohlensäure in Luft. $20^{\circ}$. Atmung mit weißem Licht kompensiert. Meßlicht $546 \mathrm{~m} \mu$. $19 \mathrm{cmm}$ Zellen im Gefäß. Absorption 13\% (Chlorophyllarme Zellen). Das Meßlicht intermittierend in $1^{\prime} 1^{\prime}$. Wir fanden $\left(v=16,59 \mathrm{~cm}^{3}, v_{\mathrm{F}}=7,00 \mathrm{~cm}^{3}\right.$, $\left.k_{02}=0,915 \mathrm{~mm}^{2}\right)$ :
1. Stde. $\mathrm{J}=9,83$
$\left.\begin{array}{l}\text { 2. Stde. } \\ \text { 3. Stde. }\end{array}\right\} \mathrm{J}=6,23$
4. Stde. $\mathrm{J}=17,7$
5. Stde. $\quad \mathrm{J}=9,83$

Druckänderungen
in mm (korr.)
11,1
15,9
17,5
5,8

\begin{tabular}{|c|c|}
\hline Perioden & $1 / q$ \\
\hline $60^{\prime}$ & 3,8 \\
\hline $120^{\prime}$ & 3,4 \\
\hline $\begin{array}{l}60^{\prime} \\
30^{\prime}\end{array}$ & $\begin{array}{l}3,8 \\
3,6\end{array}$ \\
\hline
\end{tabular}

wobei die gesamte Versuchsdauer, einschließlich der 4 dażwischen gelegten $30^{\prime}$-Perioden ohne Meßlicht, $6^{1} / 2$ Stdn. betrug und kein Abfall der Ausbeute festzustellen war.

Rechnen wir das Mittel der $1 / \varphi$-Werte mit dem Faktor 1,25 von 2,15 auf $5 \% \mathrm{CO}_{2}$ um, so erhalten wir

$$
\text { für } 5 \% \mathrm{CO}_{2} \quad 1 / \varphi=3,66 / 1,25=2,92\left[h v / \mathrm{O}_{2}\right] \text {. }
$$

2. Chlorella wurde aus der Kulturlösung zentrifugiert. Die eine Hälfte wurde mit Carbonatgemisch Nr. $9(\mathrm{~m} / 10$ 85/15) übergossen, die andere mit dem neuen Carbonatgemisch $(m / 595 / 5)$, dem die beiden Kultursalze wie in Beisp. 1 zugesetzt waren: Gasraum Luft für das alte und $2,15 \% \mathrm{CO}_{2}$-Luft für das neue Gemisch. $20^{\circ}$. Atmung mit weißem Licht kompensiert. Meßlicht $546 \mathrm{~m} \mu .22 \mathrm{cmm}$ Zellen im Gefäß. Absorption 22\% (chlorophyllreichere Zellen). Das Meßlicht intermittierend in $1^{\prime} 1^{\prime}\left(v=16,59 \mathrm{~cm}^{3}\right.$, $\left.v_{\mathrm{F}}=7,00 \mathrm{~cm}^{3}, k_{\mathrm{O} 2}=0,915 \mathrm{~mm}^{2}\right)$. Wir fanden:

in Carbonatgemisch Nr. 9 in dem neuen Gemisch 5 Stdn. $J=4,92+25 \mathrm{~mm} \quad+51 \mathrm{~mm}$ (korr.)

$$
\begin{array}{cc}
1 / \varphi \stackrel{\downarrow}{=} \mathbf{7 , 1} & \stackrel{\downarrow}{=} \mathbf{3 , 5} \\
\text { umgerechnet auf } 5 \% \mathrm{CO}_{2} \\
1 / \varphi=2,8,
\end{array}
$$

wobei zu beachten ist, daß die in dem Carbonatgemisch Nr. 9 erhaltenen Drucke nicht mit dem Faktor 1,05 korrigiert werden dürfen, da die Retention der Kohlensäure in dem Gemisch Nr. 9 fast vollständig ist.

3. Dies ist ein Versuch, in dem die maximale Ausbeute nur bei der niedrigen Intensität $J=4,98$ erhalten wurde. Es scheint uns, daß dies mit dem höheren Chlorophyllgehalt zusammenhängt. Je reicher an Chlorophyll die Zellen waren, um so niedriger mußte die Intensität sein, bei der die maximale Ausbeute erreicht wurde, was zu erwarten ist. Denn je höher der Chlorophyllgehalt ist, um so weniger Zellen beteiligen sich an der Absorption einer gegebenen Lichtmenge einer gegebenen Wellenlänge.

Zentrifugierte, nicht gewaschene Chlorella wurde mit $m / 595 / 5$ übergossen, dem die Kultursalze wie in den Beispielen 1 und 2 zugesetzt waren. Gasraum 2,15\% Kohlensäure in Luft. $20^{\circ}$. Atmung durch weißes Licht kompensiert. Meßlicht $546 \mathrm{~m} \mu .24 \mathrm{cmm}$ Zellen im Gefäß in $v_{\mathrm{F}} 7$. Absorption $29 \%$. Meßlicht intermittierend in $1^{\prime} 1^{\prime}$. Wir fanden $\left(v=16,59 \mathrm{~cm}^{3}, v_{\mathrm{F}}=7,0 \mathrm{~cm}^{3}, k_{\mathrm{O}_{2}}=0,915 \mathrm{~mm}^{2}\right)$ :

Druckänderungen
in mm(korr.) $\quad \begin{gathered}1 / \varphi=h v / \mathrm{O}_{2} \\ \text { in } 5 \% \\ 0\end{gathered}$
1. Stde. $J=19,6$
$+24,2$
2. Stde. $J=9,83$
$+18,0$
3. Stde. $J=4,98$
$+12,2$
4. Stde. $J=4,94$
$+12,2$

$\mathbf{6 , 2}$
$\mathbf{4 , 2}$
$\mathbf{3 , 1}$
$\mathbf{3 , 1}$

